\title{
Dynamical evolution of near-Sun objects
}

\author{
Vacheslav V. Emel'yanenko ${ }^{1}$ and Mikhail A. Shelyakov ${ }^{1}$ \\ ${ }^{1}$ Institute of Astronomy, Russian Academy of Sciences, \\ 48 Pyatnitskaya str., 119017, Moscow, Russia \\ email: vvemel@inasan.ru, mshelyakov@inasan.ru
}

\begin{abstract}
The dynamical evolution of short-period objects having perihelia at small heliocentric distances is discussed. We have investigated the motion of multiple-apparition members of the Marsden and Kracht sungrazing groups. The orbital evolution of these objects on timescales $<10 \mathrm{Kyr}$ is mainly determined by the Kozai-Lidov secular perturbations. These objects are dynamically connected with high-inclination near-Earth objects. On the other hand, we have found several observed near-Earth objects that evolve in the same way, reaching small perihelion distances on short timescales in the past .
\end{abstract}

Keywords. comets: sungrazing, dynamical evolution, near-Earth objects

\section{Introduction}

It is well known that near-Earth objects evolve frequently to orbits with small perihelion distances (Farinella et al. 1994, Gladman et al. 2000, Foschini et al. 2000, Marchi et al. 2009). It is estimated that up to $\sim 70 \%$ of near-Earth objects collide with the Sun during their orbital evolution (Marchi et al. 2009). The solar tide, thermal stresses and interaction with the solar atmosphere are expected to be severe for objects passing near the Sun.

Modification of the surface composition at past sungrazing conditions may be recorded in spectral reflectance properties of some existing near-Earth asteroids. But only a few very rare cases (e.g., comet P96/Machholz, in 1 Kyr (Bailey et al. 1992); 2004 LG, in 3.5 Kyr (Vokrouhlicky \& Nesvorny 2012)) have definite predictions about solar encounters of real objects in the past. All objects detected as asteroids had perihelion distances $q>0.07 \mathrm{AU}$ at the moment of discovery. However, many comets have been observed near the Sun, and some of them were registered in a few apparitions. These multipleapparition objects are usually called sunskirting comets (their perihelion distances equal $\sim 0.05 \mathrm{AU})$.

In this paper, we discuss the dynamical connection between these periodic near-Sun comets and typical near-Earth objects. We show that sunskirting comets evolve gradually to orbits with larger perihelion distances that are inherent to observed near-Earth objects. On the other hand, we have found several real near-Earth objects that were near the Sun in the recent past.

\section{Dynamical evolution of sunskirting comets}

We determined orbits of eight multiple-apparition sunskirting comets with the best observational material ( 2 members of the Kracht group, 1 member of the Kracht 2 group and 5 members of the Marsden group) (Emel'yanenko \& Shelyakov 2014). Two of them have observations in three apparitions, but no significant nongravitational effects were found in our calculations. To study the dynamical evolution of these objects we considered 


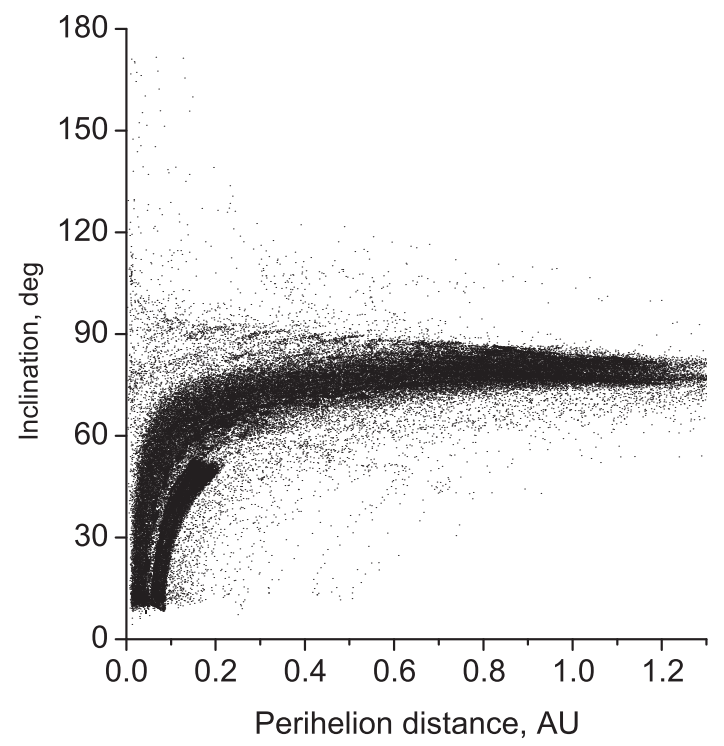

Figure 1. Changes of perihelion distances and inclinations for sunskirting objects. Data for orbits from the confidence region are plotted every 100 years.

a set of 200 initial orbits from the confidence region for each object, using the covariance matrix. These orbits were integrated forward taking account of perturbations from all planets. The dynamical evolution of test particles was calculated using the symplectic integrator (Emel'yanenko 2007). Particles were removed from integrations when they collide with planets or $q<0.005 \mathrm{AU}$ or the semimajor axis $a>50 \mathrm{AU}$.

Fig. 1 shows the changes of perihelion distances and inclinations for the studied objects for 10000 years. The planetary perturbations lead to drastic changes of orbits on a relatively short interval of time. The black region in Fig. 1 corresponds to those orbits which are the most probable for the former sunskirting objects in the future.

The systematic increase in perihelion distances and inclinations is caused by the LidovKozai secular perturbations. Fig. 2 shows the evolution of the semimajor axis, eccentricity, inclination and argument of perihelion for the sungrazing object C/2004 R4 = C/2007 Y4, representing a typical example of the Lidov-Kozai circles for changes of orbital elements.

\section{Recent approaches of observed asteroids to the Sun}

The results on the dynamical evolution of sunskirting comets (Fig. 1) show that the present high-inclination near-Earth objects may have experienced close approaches to the Sun in the recent past. To analyze this question, we integrated back orbits of several observed high-inclination near-Earth objects. We applied the same numerical methods as above for sunskirting comets. Some remarkable examples of asteroids with recent passages near the Sun are shown in Fig. 3, 4. 2003 EH1 had $q \sim 0.09$ AU 1.4 Kyr ago. 2012 FZ23 had $q \sim 0.06$ AU $2.8 \mathrm{Kyr}$ ago. We have found also other observed near-Earth objects that had definitely solar encounters in the recent past $(2010 \mathrm{KY} 27, q \sim 0.08 \mathrm{AU}$ in $0.8 \mathrm{Kyr} ; 2001 \mathrm{AU} 43, q \sim 0.12 \mathrm{AU}$ in $7.9 \mathrm{Kyr} ; 2008 \mathrm{GV} 3, q \sim 0.12 \mathrm{AU}$ in $9.0 \mathrm{Kyr})$.

It is natural to assume that the solar tide, thermal stresses and interaction with the solar atmosphere could lead to disruption and surface modification of bodies near the Sun. This may be important in determining the population of near-Earth objects. Unfortunately, there are no direct observational indications how to distinguish objects 

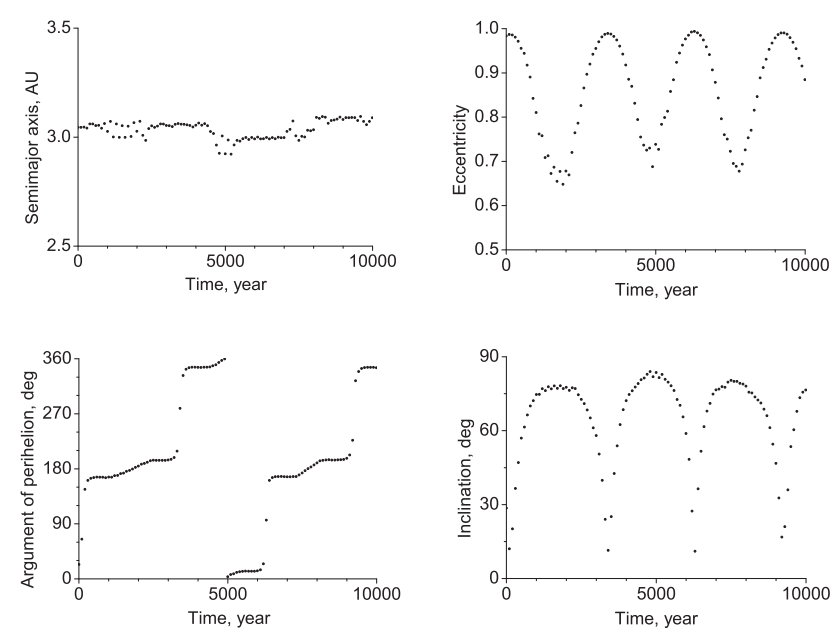

Figure 2. Changes of the semimajor axis, eccentricity, inclination and argument of perihelion for the sungrazing object $\mathrm{C} / 2004 \mathrm{R} 4=\mathrm{C} / 2007 \mathrm{Y} 4$. Data are plotted every 100 years.

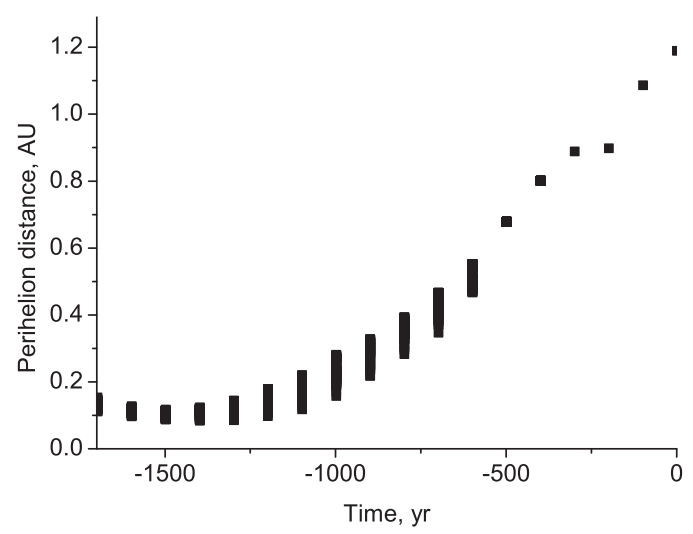

Figure 3. Changes of $q$ for 2003 EH1. Data for orbits from the confidence region are plotted every 100 year.

that have visited the near-Sun region. In particular, Jewitt (2013) concluded that his observations provided no evidence to suggest that physical properties of small-perihelion objects were measurably influenced by the heat of the Sun.

We hope that our results on new observed near-Earth objects, moving in orbits with small perihelion distances in the recent past, will stimulate additional investigations in this direction. Special observations of these objects are very desirable.

\section{Conclusions}

1. The orbital evolution of the Kracht and Marsden group members on timescales $<10$ Kyr is mainly determined by the Kozai-Lidov secular perturbations. These objects are dynamically connected with high-inclination near-Earth objects.

2. We have found several observed near-Earth objects that evolve in the same way, reaching small perihelion distances on short timescales in the past. 


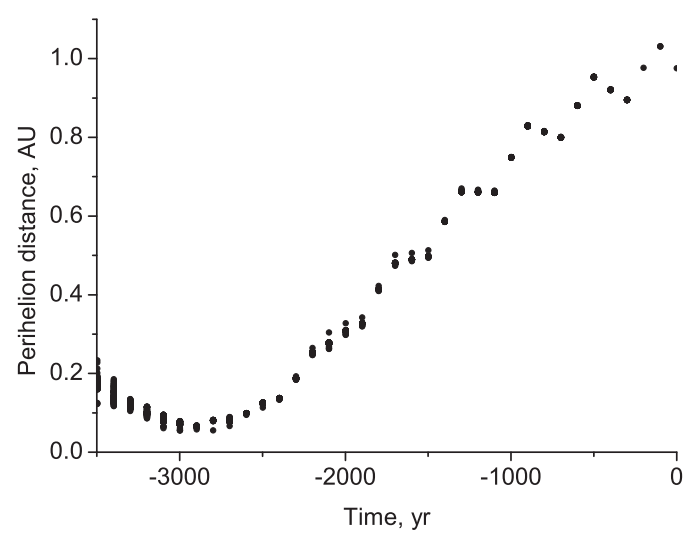

Figure 4. Changes of $q$ for 2012 FZ23. Data for orbits from the confidence region are plotted every 100 years.

\section{References}

Bailey, M. E., Chambers, J. E., \& Hahn, G. 1992, A\& A, 257, 315

Emel'yanenko 2007, Celestial Mechanics \& Dynamical Astronomy, 98, 191

Emel'yanenko, V. V. \& Shelyakov, M. A. 2014, Icarus, submitted

Farinella, P., Froeschle, Ch., Froeschle, Cl., Gonczi, R., Hahn, G., Morbidelli, A., \& Valsecchi, G. B. 1994, Nature, 371, 314

Foschini, L., Farinella, P., Froeschle, Ch., Gonczi, R., Jopek, T. J., \& Michel, P. 2000, A\&̛A, 353, 797

Gladman, B., Michel, P., \& Froeschle, Ch. 2000, Icarus, 146, 176

Jewitt 2013, AJ, 145, 133

Marchi, S., Delbo, M., Morbidelli, A., Paolicchi, P., \& Lazzarin, M. 2009, MNRAS, 400, 147

Vokrouchlicky, D. \& Nesvorny, D. 2012, A\&A, 541, A109 